\title{
Garlic consumption and colorectal cancer risk in man: a systematic review and meta-analysis
}

\author{
Manuela Chiavarini ${ }^{1}$, Liliana Minelli ${ }^{1}$ and Roberto Fabiani ${ }^{2, *}$ \\ 'Department of Experimental Medicine, Section of Public Heath, University of Perugia, Perugia, Italy: ${ }^{2}$ Department of \\ Chemistry, Biology and Biotechnology, University of Perugia, Via del Giochetto, 06126 Perugia, Italy
}

Submitted 14 May 2014: Final revision received 29 January 2015: Accepted 25 February 2015: First published online 6 May 2015

\begin{abstract}
Objective: Colorectal cancer shows large incidence variations worldwide that have been attributed to different dietary factors. We conducted a meta-analysis on the relationship between garlic consumption and colorectal cancer risk.

Design: We systematically reviewed publications obtained by searching ISI Web of Knowledge, MEDLINE and EMBASE literature databases. We extracted the risk estimate of the highest and the lowest reported categories of intake from each study and conducted meta-analysis using a random-effects model.

Results: The pooled analysis of all fourteen studies, seven cohort and seven casecontrol, indicated that garlic consumption was not associated with colorectal cancer risk $\left(\mathrm{OR}=0.93 ; 95 \%\right.$ CI $\left.0.82,1.06, P=0.281 ; I^{2}=83.6 \%, P \leq 0.001\right)$. Separate analyses on the basis of cancer sites and sex also revealed no statistically significant effects on cancer risk. However, when separately analysed on the basis of study type, we found that garlic was associated with an approximately $37 \%$ reduction in colorectal cancer risk in the case-control studies (combined risk estimate $=0.63,95 \%$ CI $0.48,0.82, P=0.001 ; I^{2}=75.6 \%, P \leq 0.001$ ).

Conclusions: Our results suggest that consumption of garlic is not associated with a reduced colorectal cancer risk. Further investigations are necessary to clarify the discrepancy between results obtained from different types of epidemiological studies.
\end{abstract}

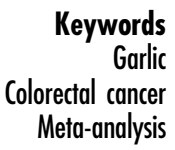

Colorectal cancer (CRC) is the third most common cancer worldwide, with an estimated number of new cases and deaths in 2008 of 1.2 million and 608000 (accounting for $8 \%$ of all cancer deaths), respectively ${ }^{(1)}$. CRC incidence rates vary markedly worldwide, with rates per 100000 from 4.1 to 59.1 for males and from 3.6 to 39.5 for females $^{(2)}$. These large variations have been attributed to different environmental, lifestyle and dietary factors. In particular, diet and nutrition are estimated to explain as much as $50 \%$ of the worldwide incidence of $\mathrm{CRC}^{(3)}$.

Among different foods, garlic (Allium satioum) has received a particular attention in recent years because of its high content of organosulfur compounds and flavonoids ${ }^{(4)}$. The allyl sulfur constituents in garlic, which comprise $~ 1 \%$ of its dry weight, seem to be responsible for its chemopreventive effects $^{(5)}$. In particular, these compounds have been shown to reduce the formation of aberrant crypt foci and to prevent carcinogen-induced colon cancer in different animal models ${ }^{(6)}$. The mechanisms by which sulfur compounds inhibit cancer cell growth have been the topic of intense research over the last two decades and include: activation of metabolizing enzymes that detoxify carcinogens; reduction of DNA adducts; antioxidant effects; regulation of cellcycle arrest; induction of apoptosis and differentiation; histone modification; and inhibition of angiogenesis and invasion $^{(7,8)}$. On the other hand, conclusions from epidemiological research aimed to find an association between garlic consumption and $\mathrm{CRC}$ risk are inconsistent or even contradictory ${ }^{(9,10)}$. In a previous meta-analysis, a risk reduction of $31 \%$ could be observed between CRC and garlic intake (raw and cooked, excluding garlic supplements; RR $=0.69 ; 95 \%$ CI $0.55,0.89)^{(11)}$. Since then several other studies have been published on this topic with contrasting results. Therefore we conducted a meta-analysis for deriving a more precise estimation of this association.

\section{Materials and methods}

\section{Search strategy}

Our literature search was aimed at identifying available research studies that examined the effects of garlic on 
CRC. Studies included in our meta-analysis were identified by searching, without restrictions, multiple literature databases including ISI Web of Knowledge, MEDLINE and EMBASE, and selecting all articles published up to September 2014. The following strings were used for the search: ('garlic' OR 'allium sativum') AND ('cancer' OR 'neoplastic disease' OR 'neoplasm') AND ('colon' OR 'colorectal' OR 'rectal'). Additionally, we also checked the reference lists of retrieved papers and recent reviews. After removing duplicates in the primary research we identified 439 studies. Although useful to have background information, reviews and meta-analyses were excluded.

\section{Data collection}

We systematically reviewed and selected the studies that met all of the following criteria: (i) the study (cohort or case-control) must have had garlic consumption assessed; (ii) it must have provided a risk estimate (hazard ratio, relative risk or odds ratio) for colorectal, colon or rectal cancer incidence as well as its 95\% confidence interval; and (iii) it must have provided information on adjustment for confounding factors. Two investigators reviewed the eligibility of all studies according to the predetermined selection criteria independently. From the results of the selected studies, we extracted the risk estimate of the highest relative to the lowest intake for the analysis. For the overall estimation the hazard ratio and relative risk were taken to be approximations to the odds ratio, and the meta-analysis was done as if all types of ratio were odds ratios. The combined risk estimate was calculated using a random-effects model in which the effect measures were odds ratios or relative risks. In this analysis data from both females and males, and from colon, rectal and colorectal cancer, as independent populations, were included.

\section{Assessment of study quality}

The study quality was assessed by a system based on the Newcastle-Ottawa Scale method ${ }^{(12)}$. Two investigators (R.F. and M.C.) assessed the quality of each selected study and discrepancies were addressed by a joint re-evaluation of the original article with a third reviewer. The full score was 9 and a total score $\geq 7$ was used to indicate a highquality study. To avoid selection bias, no study was excluded because of these quality criteria.

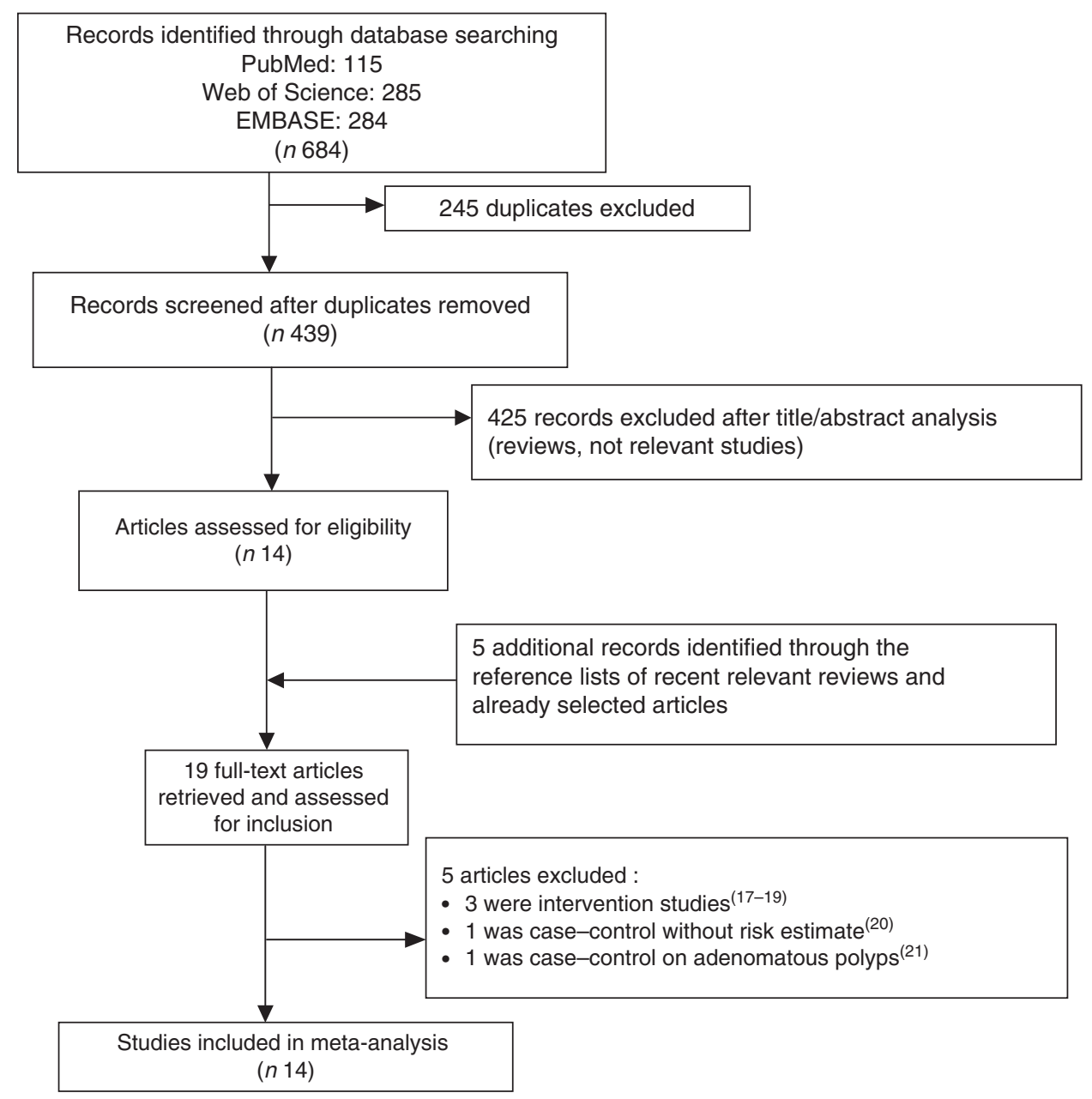

Fig. 1 Flowchart of the study selection process 


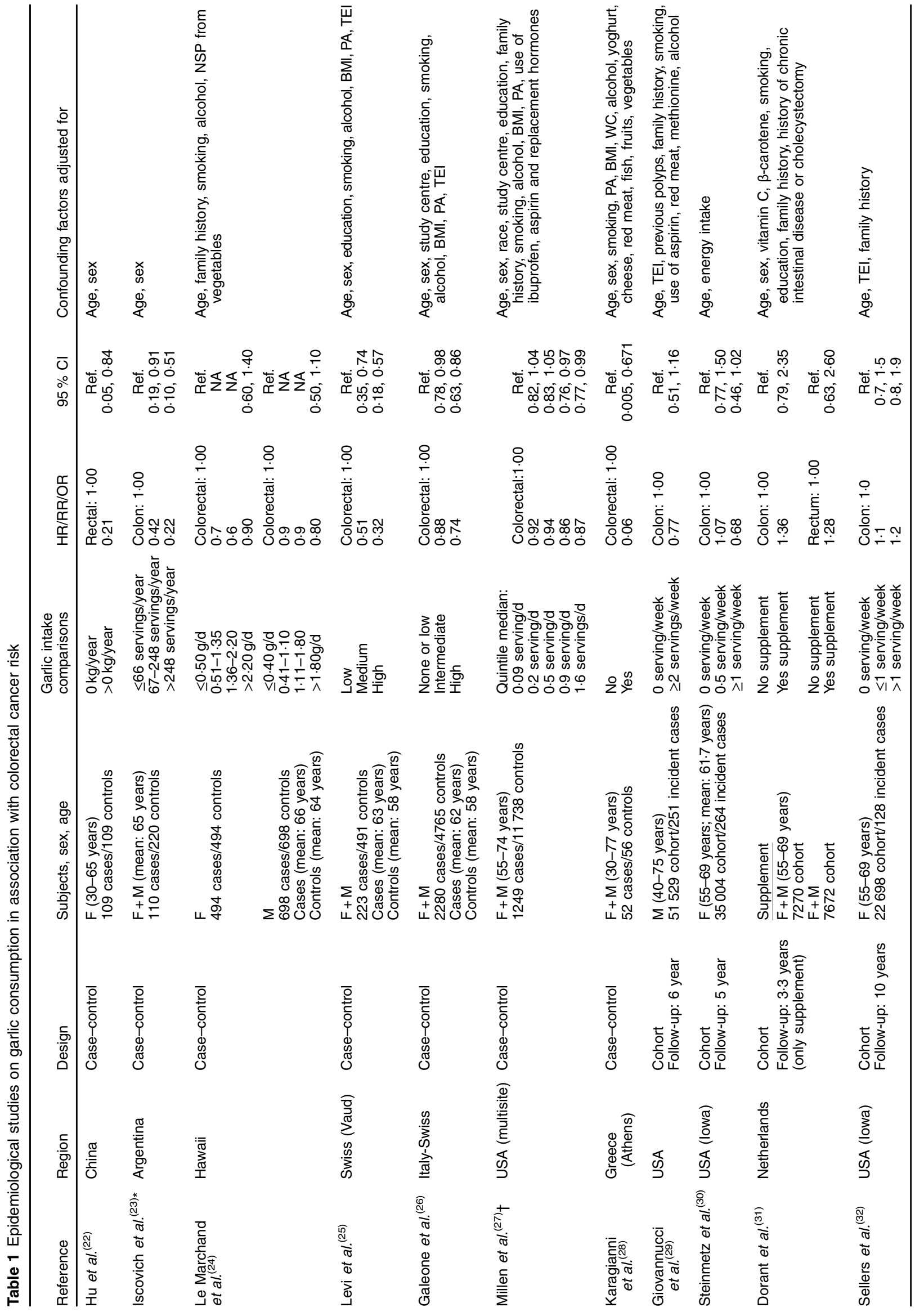




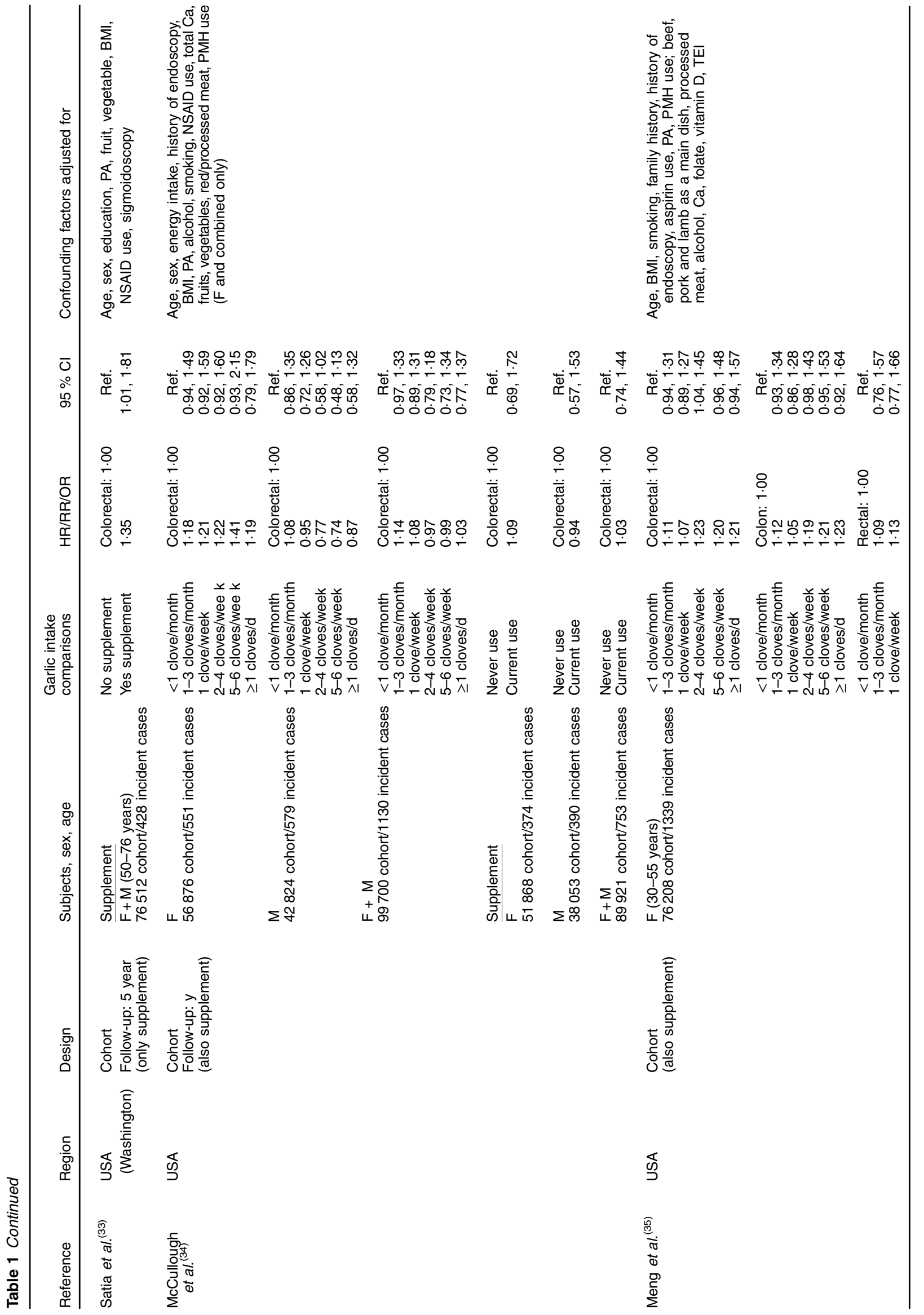




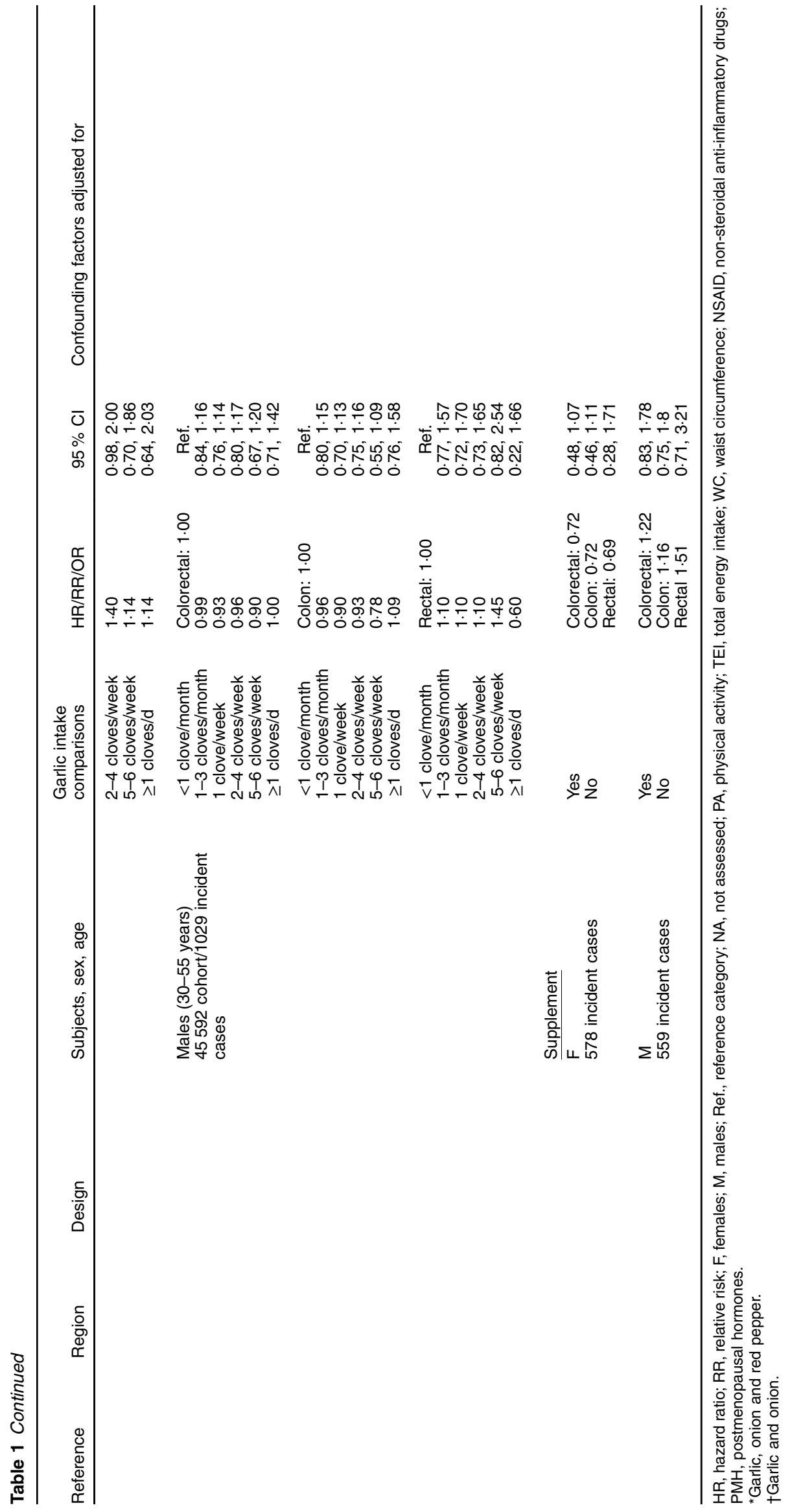




\section{Statistical examination}

Heterogeneity between studies was assessed using the Cochrane $Q$ test and $I^{2}$ score. The $\chi^{2}$-based Cochran's $Q$ statistic and the $I^{2}$ statistic were used to quantify evaluated heterogeneity ${ }^{(13)}$. The $I^{2}$ statistic yields results ranging from 0 to $100 \%$ and $I^{2}>50 \%$ represents substantial heterogeneity ${ }^{(14)}$. Results of the meta-analysis may be biased if the probability of a study being published is dependent on its results. We used the methods of Begg and Mazumdar ${ }^{(15)}$ and Egger et $a l^{(16)}$ to detect publication bias. Both methods test for funnel plot asymmetry, the former being based on the rank correlation between the effect estimates and their sampling variances, and the latter on a linear regression of a standard normal deviate on its precision. If a potential bias was detected, we further conducted a sensitivity analysis to assess the robustness of combined effect estimates and the possible influence of the bias and to have the bias corrected. We also conducted a sensitivity analysis to investigate the influence of each single study on the overall risk estimate by omitting one study in turn. We considered the funnel plot to be asymmetrical if the intercept of Egger's regression line deviated from zero with a $P$ value of less than $0 \cdot 10$. We should note that this test for asymmetry possesses relatively low power to detect a real publication bias when the total number of studies included in the meta-analysis is small (twenty-five or fewer), which is the case in the current review. The ProMeta Version 2.0 statistical program (Internovi) was used for the analysis. All reported $P$ values are from two-sided statistical tests and differences with $P \leq 0.05$ were considered significant.

\section{Results}

The flowchart of the study selection process is shown in Fig. 1. After the analysis of titles and abstracts, we identified fourteen studies on garlic consumption and CRC risk in man. In addition, five studies, identified through the reference lists of recent relevant reviews and already selected articles, were included for the analysis. After the full-text assessment five studies were excluded from the analysis as follows: three were intervention trials that tested the effect of aged garlic extracts on colorectal adenoma occurrence ${ }^{(17-19)}$, one was a case-control study not reporting the risk estimate ${ }^{(20)}$ and one was a casecontrol study on adenomatous polyps ${ }^{(21)}$ (Fig. 1). Therefore only fourteen studies met the inclusion criteria: seven were case-control ${ }^{(22-28)}$ and seven were cohort studies $^{(29-35)}$ (Table 1). Among the cohort studies two considered garlic supplements only ${ }^{(31,33)}$, while among the other five studies two considered garlic supplements in addition to dietary intake $\mathrm{e}^{(34,35)}$. Table 1 summarizes the detailed characteristics of included studies. Three studies reported findings only for females ${ }^{(22,30,32)}$, one only for males ${ }^{(29)}$, eight for females and males together ${ }^{(23,25-28,31,33,34)}$ and three presented findings for males and females separately ${ }^{(24,34,35)}$. Three studies reported results of risk for rectal cancer ${ }^{(22,31,35)}$, six for colon cancer $^{(23,29-32,35)}$ and eight for $\mathrm{CRC}^{(24-28,33-35)}$.

Study-specific quality scores of each study are summarized in Supplemental Table S1 and Supplemental Table S2 for case-control and cohort studies, respectively (see online supplementary material). The ranges of quality score were from 5 to 8 (median: 6) and from 6 to 9 (median: 8) for case-control and cohort studies, respectively. High-quality studies (i.e. those studies that had a score $\geq 7$ ) included three case-control $^{(26-28)}$ and six cohort ${ }^{(29-34)}$ studies.

The analysis of the fourteen studies pooled together yielded a combined risk estimate of 0.93 (95\% CI 0.82 , 1.06; $P=0 \cdot 281)$ and test of heterogeneity $Q=176 \cdot 85\left(I^{2}=\right.$ $83.6 \%, P \leq 0.001)$. Publication bias was investigated by a funnel plot (Fig. 2). Bias detection revealed a significant effect $(P \leq 0 \cdot 001)$ using the method of Begg and Mazum$\operatorname{der}^{(15)}$, while no bias was detected by the Egger test ${ }^{(16)}$ $(P=0 \cdot 121)$. Sensitivity analyses investigating the influence of each single study on the overall risk estimate by omitting one study in turn suggested that the overall risk estimates were not substantially modified by any single study, with a range from $0.87(95 \% \mathrm{CI} 0.73,1.04)$ to 0.96 (95\% CI $0 \cdot 84,1 \cdot 08)$. Of note, the heterogeneity was still observed after omitting each study in turn. Further analyses were performed by stratifying the data on the basis of study type. The forest plots are reported in Fig. 3(a) (casecontrol studies), Fig. 3(b) (cohort studies) and Fig. 3(c) (supplement studies). The results showed that only in the case-control studies was there a statistically significant reduction $(37 \%)$ of cancer risk in association with garlic intake, with a risk estimate of 0.63 (95\% CI $0.48,0.82$; $P=0 \cdot 001$; Table 2). Table 2 also reports the results of both heterogeneity and publication bias tests. For the casecontrol studies only, a high significant heterogeneity was observed while publication bias was significant by the Egger test. A sensitivity analysis excluding Karagianni et al.'s study ${ }^{(24)}$, which caused asymmetry of the funnel plot, yielded a combined risk estimate of 0.65 (95\% CI

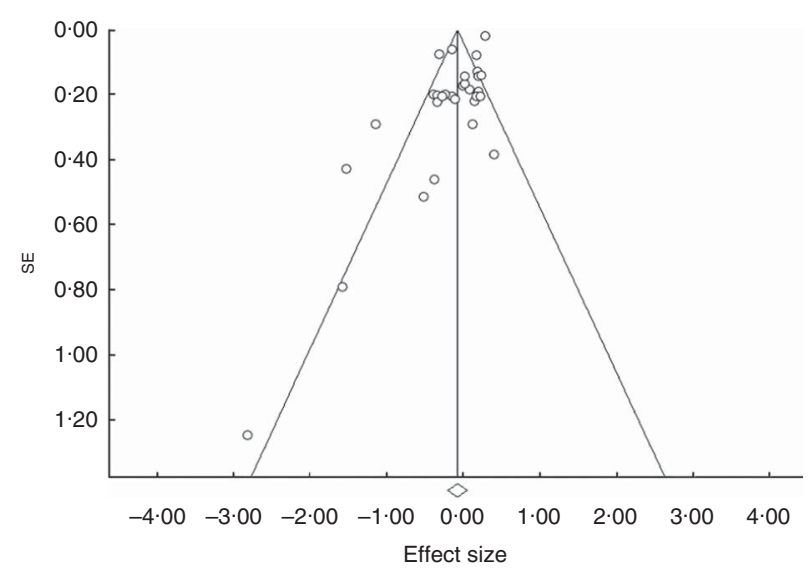

Fig. 2 Funnel plot for the association between garlic consumption and colorectal cancer 
(a)

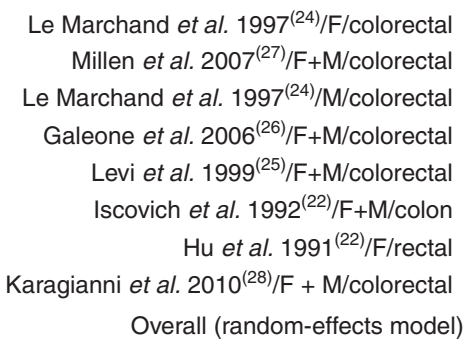

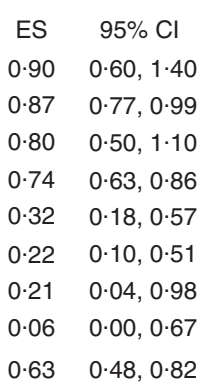

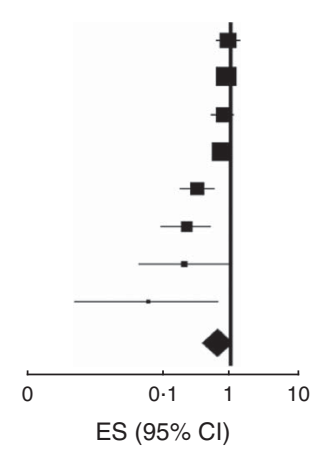

(b) Meng et al. $2013^{(35)} / \mathrm{F} /$ colon Meng et al. $2013^{(35)} / \mathrm{F} /$ colorectal Sellers et al. 1998 $8^{(32)} / \mathrm{F} /$ colon McCullough et al. 2012 ${ }^{(34)} / \mathrm{M} /$ colorectal Meng et al. $2013^{(35)} / \mathrm{F} / \mathrm{rectal}$ Meng et al. $2013^{(35)} / \mathrm{M} / \mathrm{colon}$ McCullough et al. 2012 ${ }^{(34)} / \mathrm{F}+\mathrm{M} /$ colorectal Meng et al. $2013^{(35)} / \mathrm{M} /$ colorectal McCullough et al. 2012(34)/F/colorectal Giovannucci et al. 1994 $4^{(29)} / \mathrm{M} /$ colon Steinmetz et al. $1994^{(30)} / \mathrm{F} /$ colon Meng et al. $2013^{(35)} / \mathrm{M} /$ rectal Overall (random-effects model)

$\begin{array}{cc}\text { ES } & 95 \% \mathrm{Cl} \\ 1 \cdot 23 & 0 \cdot 92,1 \cdot 64 \\ 1 \cdot 21 & 0 \cdot 94,1 \cdot 57 \\ 1 \cdot 20 & 0 \cdot 80,1 \cdot 90 \\ 1 \cdot 19 & 0 \cdot 79,1 \cdot 79 \\ 1 \cdot 14 & 0 \cdot 64,2 \cdot 03 \\ 1 \cdot 09 & 0 \cdot 76,1 \cdot 58 \\ 1 \cdot 03 & 0 \cdot 77,1 \cdot 37 \\ 1.00 & 0 \cdot 71,1 \cdot 42 \\ 0.87 & 0 \cdot 58,1 \cdot 32 \\ 0.77 & 0.51,1 \cdot 16 \\ 0.68 & 0 \cdot 46,1 \cdot 02 \\ 0.60 & 0 \cdot 22,1 \cdot 66 \\ 1.04 & 0 \cdot 93,1 \cdot 16\end{array}$

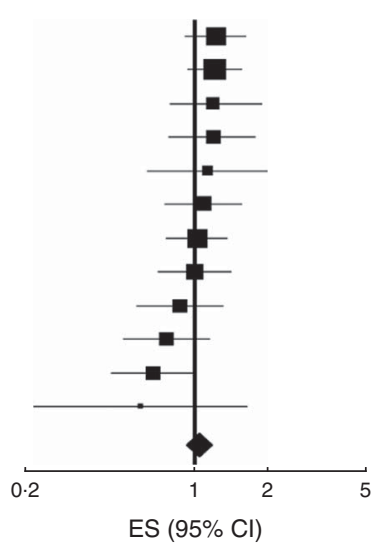

(c)

Meng et al. 2013 ${ }^{(35)} /$ supplement/M/rectal Satia et al. $2009^{(33)} / \mathrm{F}+\mathrm{M} /$ colorecta Dorant et al. $1996^{(31)} / \mathrm{F}+\mathrm{M} /$ recta Dorant et al. 1996 ${ }^{(31)} / \mathrm{F}+\mathrm{M} /$ colon Meng et al. $2013^{(35)} /$ supplement/M/colorectal Meng et al. $2013^{(35)} /$ supplement/M/colon McCullough et al. $2012^{(34)}$ supplement/F+M/colorectal Meng et al. $2013^{(35)} /$ supplement/F/colon Meng et al. $2013^{(35)} /$ supplement/F/colorectal Meng et al. $2013^{(35)} /$ supplement/F/recta Overall (random-effects model)

$\begin{array}{cc}\text { ES } & 95 \% \mathrm{Cl} \\ 1.51 & 0.71,3.21 \\ 1.35 & 1.01,1.81 \\ 1.28 & 0.63,2.60 \\ 1.26 & 0.84,1.91 \\ 1.22 & 0.83,1.78 \\ 1.16 & 0.75,1.80 \\ 1.03 & 0.74,1.44 \\ 0.72 & 0.46,1.11 \\ 0.72 & 0.48,1.07 \\ 0.69 & 0.28,1.71 \\ 1.07 & 0.91,1.26\end{array}$

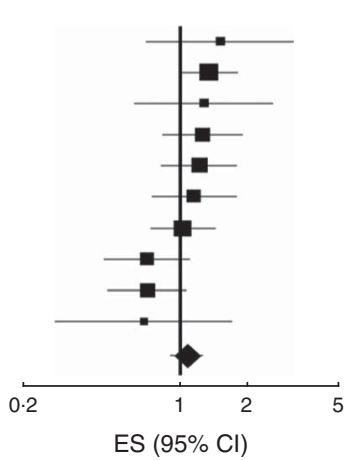

Fig. 3 Forest plot for the association between garlic consumption and colorectal cancer by study design: (a) case-control, (b) cohort and (c) supplement studies. The study-specific effect size (ES) and $95 \% \mathrm{Cl}$ are represented by the black square and horizontal line, respectively; the area of the black square is proportional to the specific-study weight to the overall meta-analysis. The centre of the diamond presents the pooled RR risk and its width represents the pooled $95 \% \mathrm{Cl}$ ( $\mathrm{F}$, females; M, males)

$0 \cdot 51,0 \cdot 84 ; P \leq 0 \cdot 01)$ with $Q=24 \cdot 45\left(I^{2}=75.5 \%, P \leq 0 \cdot 001\right)$, and $P=0.054$ and $P=0.176$ for publication bias by the Begg and Egger methods, respectively. We further separately analysed studies according to cancer sites (colon, rectum, colorectal) and sex (female, male, both). No significant effects were observed in all cases (Table 2).

\section{Discussion}

The results of the current meta-analysis indicate that, when all selected studies ( $n$ 14) were considered, garlic consumption was not associated with CRC risk. A small reduction of risk was observed (7\%) but this effect was not statistically significant. Our results are in contrast with a previous meta-analysis based on seven studies, four casecontrol $^{(22-25)}$ and three cohort $^{(29-31)}$, which suggested a preventive effect and estimated that high consumption of garlic decreases the risk of CRC by $30 \%{ }^{(11)}$. In comparison with the previous meta-analysis, our updated search identified and included seven more studies ${ }^{(26-28,32-35)}$ which may be responsible for these discrepancies. In addition, our analysis included the estimated risks associated with the use of garlic supplements which were excluded in the previous 
meta-analysis ${ }^{(11)}$. Stratification of the sample on the basis of cancer sites (colon, rectal and colorectal) and sex (female, male and both) also revealed no statistically significant effects on CRC risk. However, when separately analysed on the basis of study type, we found that garlic was associated with a significant reduction (37\%) of CRC risk in the casecontrol studies while no effects were observed for both cohort and supplement studies. This discrepancy between the case-control and cohort studies may be due to several reasons. Case-control studies have several weaknesses and critical points, which can lead to incorrect conclusions. They are particularly susceptible to recall and selection bias which may produce misclassification of exposure between case and control groups. Moreover, the control group may not be representative of the general population as a consequence of various degrees of selection bias among healthy subjects ${ }^{(36)}$. In addition, some case-control studies included in the current meta-analysis did not adjust for important confounding factors such as red meat, energy intake, alcohol and others that have been consistently associated with CRC risk $^{(37)}$. Therefore, as discussed above, findings derived from retrospective studies should be interpreted with caution while cohort studies certainly have a greater degree of reliability. However, it should be noted that six cohort studies out of seven were performed in the USA, so suggesting that geographical differences may exist. Similarly to cohort studies, also the results on supplements suggest that use of garlic supplements was not able to prevent CRC. Our results are in agreement with a recent meta-analysis, published during the preparation of this manuscript, investigating the association between high intake of allium vegetables and CRC risk ${ }^{(38)}$. In that study, the stratified analysis showed a not statistically significant increase of CRC risk in association with garlic intake in cohort studies (OR $=1 \cdot 11 ; 95 \%$ CI $0.95,1.29)^{(38)}$. In addition, the use of garlic supplements was associated with a significant increase of CRC risk $(\mathrm{OR}=1 \cdot 18 ; 95 \% \mathrm{CI} 1 \cdot 02,1 \cdot 36)^{(38)}$. The small difference with our data may be due to the inclusion in our analysis of two studies $^{(29,30)}$ which were excluded in the above reported meta-analysis ${ }^{(38)}$. Moreover, in the analysis on supplements we used a random-effects model and excluded data from past use of garlic supplements ${ }^{(34)}$.

Recently, two meta-analyses have been published showing the effects of garlic intake on gastric ${ }^{(39)}$ and prostate cancer risk ${ }^{(40)}$. Similarly to our results, a preventive effect of garlic on gastric cancer was observed in three hospital-based case-control studies (OR $=0.57 ; 95 \%$ CI 0.34, $0 \cdot 80)$ and in eight population-based case-control studies $(\mathrm{OR}=0.52 ; 95 \% \mathrm{CI} 0.37,0.67)$, while one cohort study showed an increased risk $(\mathrm{OR}=1 \cdot 28 ; 95 \% \text { CI } 0 \cdot 45,3 \cdot 66)^{(39)}$. Furthermore, the results obtained on prostate cancer were similar to our results in showing that meta-analysis from the case-control studies suggested a significant reduction in risk $(\mathrm{OR}=0.77 ; 95 \% \mathrm{CI} 0.64,0.91)$ while the results from the cohort studies were null $(\mathrm{OR}=0.96 ; 95 \% \mathrm{CI} 0.89,1.05)^{(40)}$. Several mechanisms have been suggested to participate in 
the potential anticancer effects of garlic and its components. Garlic is rich in organosulfur compounds and flavonoids, which have been reported to exert chemopreventive effects in animal and in vitro studies by different mechanisms including modulation of carcinogen-metabolizing enzymes, cell-cycle arrest, induction of apoptotic cell death and/or differentiation, suppression of oncogenic signal transduction pathways, and inhibition of neoangiogenesis ${ }^{(8,41)}$. It should be noted that all of these effects have been evidenced at high doses of compounds which may be not easily reached with the normal human diet. This is particularly evident for garlic, which is generally used in low amounts.

\section{Conclusion}

The present meta-analysis provides evidence that consumption of garlic is not associated with a reduced CRC risk. The preventive effect suggested by the case-control studies may be due to potential confounding factors and exposure misclassification. Further studies will be needed to clarify these discrepancies; in particular, cohort studies should be carried out in the continents of Asia and Europe to confirm the US findings.

\section{Acknowledgements}

Financial support: All work was completed at the University of Perugia, Italy. The authors thank their home institution for financial support. The University of Perugia had no role in the design, analysis or writing of this article. Conflict of interest: None. Authorship: Study concept and design: M.C., L.M. and R.F. Acquisition of data: M.C. and R.F. Analysis and interpretation of data: M.C., L.M. and R.F. All authors contributed substantively to this manuscript, were involved with critical revisions to the manuscript and provided approval for its publication. Ethics of buman subject participation: Ethical approval was not required.

\section{Supplementary material}

To view supplementary material for this article, please visit http://dx.doi.org/10.1017/S1368980015001263

\section{References}

1. Ferlay J, Shin HR, Bray F et al. (2010) Estimates of worldwide burden of cancer in 2008: GLOBOCAN 2008. Int J Cancer 127, 2893-2917.

2. Center MM, Jemal A, Smith RA et al. (2009) Worldwide variations in colorectal cancer. CA Cancer J Clin 59, 366-378.

3. Vargas AJ \& Thompson PA (2012) Diet and nutrient factors in colorectal cancer risk. Nutr Clin Pract 27, 613-623.
4. Iciek M, Kwiecień I \& Włodek L (2009) Biological properties of garlic and garlic-derived organosulfur compounds. Environ Mol Mutagen 50, 247-265.

5. El-Bayoumy K, Sinha R, Pinto JT et al. (2006) Cancer chemoprevention by garlic and garlic-containing sulfur and selenium compounds. J Nutr 136, 3 Suppl., S864-S869.

6. Ross SA, Finley JW \& Milner JA (2006) Allyl sulfur compounds from garlic modulate aberrant crypt formation. J Nutr 136, 3 Suppl., S852-S854.

7. Powolny AA \& Singh SV (2008) Multitargeted prevention and therapy of cancer by diallyl trisulfide and related allium vegetable-derived organosulfur compounds. Cancer Lett 269, 305-314.

8. Yi L \& Su Q (2013) Molecular mechanisms for the anticancer effects of diallyl disulfide. Food Chem Toxicol 57, 362-370.

9. Ngo SN, Williams DB, Cobiac L et al. (2007) Does garlic reduce risk of colorectal cancer? A systematic review. J Nutr 137, 2264-2269.

10. Alpers DH (2009) Garlic and its potential for prevention of colorectal cancer and other conditions. Curr Opin Gastroenterol 25, 116-121.

11. Fleischauer AT, Poole C \& Arab L (2000) Garlic consumption and cancer prevention: meta-analyses of colorectal and stomach cancers. Am J Clin Nutr 72, 1047-1052.

12. Wells GA, Shea B, O'Connell D et al. (2014) The Newcastle-Ottawa Scale (NOS) for assessing the quality of nonrandomised studies in meta-analyses. http://www.ohri. $\mathrm{ca} /$ programs/clinical_epidemiology/oxford.asp (accessed September 2014).

13. Higgins JP \& Thompson SG (2002) Quantifying heterogeneity in a meta-analysis. Stat Med 21, 1539-1558.

14. Higgins JP, Thompson SG, Deeks JJ et al. (2003) Measuring inconsistency in meta-analyses. BMJ 327, 557-560.

15. Begg CB \& Mazumdar M (1994) Operating characteristics of a rank correlation test for publication bias. Biometrics $\mathbf{5 0}$, 1088-1101.

16. Egger M, Davey Smith G, Schneider M et al. (1997) Bias in meta-analysis detected by a simple, graphical test. BMJ 315, 629-634.

17. Tanaka S, Haruma K, Kunihiro M et al. (2004) Effects of aged garlic extract (AGE) on colorectal adenomas: a doubleblinded study. Hiroshima J Med Sci 53, 39-45.

18. Tanaka S, Haruma K, Yoshihara M et al. (2006) Aged garlic extract has potential suppressive effect on colorectal adenomas in humans. $J$ Nutr 136, 3 Suppl., 821S-826S.

19. Ishikawa H, Saeki T, Otani $\mathrm{T}$ et al. (2006) Aged garlic extract prevents a decline of NK cell number and activity in patients with advanced cancer. J Nutr 136, 3 Suppl., 816S-820S.

20. Kotzev I, Mirchev M, Manevska B et al. (2008) Risk and protective factors for development of colorectal polyps and cancer (Bulgarian experience). Hepatogastroenterology $\mathbf{5 5}$, 381-387.

21. Witte JS, Longnecker MP, Bird CL et al. (1996) Relation of vegetable, fruit, and grain consumption to adenomatous polyps. Am J Epidemiol 144, 1015-1025.

22. Hu JF, Liu YY, Yu YK et al. (1991) Diet and cancer of the colon and rectum: a case-control study in China. Int $J$ Epidemiol 20, 362-367.

23. Iscovich JM, L'Abbé KA, Castelleto R et al. (1992) Colon cancer in Argentina. I: risk from intake of dietary items. Int $J$ Cancer 51, 851-857.

24. Le Marchand L, Hankin JH, Wilkens LR et al. (1997) Dietary fiber and colorectal cancer risk. Epidemiology $\mathbf{8}$, 658-665.

25. Levi F, Pasche C, La Vecchia C et al. (1999) Food groups and colorectal cancer risk. Br J Cancer 79, 1283-1287.

26. Galeone C, Pelucchi C, Levi F et al. (2006) Onion and garlic use and human cancer. Am J Clin Nutr 84, 1027-1032. 
27. Millen AE, Subar AF, Graubard BI et al. (2007) Fruit and vegetable intake and prevalence of colorectal adenoma in a cancer screening trial. Am J Clin Nutr 86, 1754-1764.

28. Karagianni V, Merikas E, Georgopoulos F et al. (2010) Risk factors for colorectal polyps: findings from a Greek casecontrol study. Rev Med Chir Soc Med Nat Iasi 114, 662-670.

29. Giovannucci E, Rimm EB, Stampfer MJ et al. (1994) Intake of fat, meat, and fiber in relation to risk of colon cancer in men. Cancer Res 54, 2390-2397.

30. Steinmetz KA, Kushi LH, Bostick RM et al. (1994) Vegetables, fruit, and colon cancer in the Iowa Women's Health Study. Am J Epidemiol 139, 1-15.

31. Dorant E, van den Brandt PA \& Goldbohm RA (1996) A prospective cohort study on the relationship between onion and leek consumption, garlic supplement use and the risk of colorectal carcinoma in The Netherlands. Carcinogenesis 17, 477-484.

32. Sellers TA, Bazyk AE, Bostick RM et al. (1998) Diet and risk of colon cancer in a large prospective study of older women: an analysis stratified on family history (Iowa, United States). Cancer Causes Control 9, 357-367.

33. Satia JA, Littman A, Slatore CG et al. (2009) Associations of herbal and specialty supplements with lung and colorectal cancer risk in the VITamins and Lifestyle study. Cancer Epidemiol Biomarkers Prev 18, 1419-1428.
34. McCullough ML, Jacobs EJ, Shah R et al. (2012) Garlic consumption and colorectal cancer risk in the CPS-II Nutrition Cohort. Cancer Causes Control 23, 1643-1651.

35. Meng S, Zhang X, Giovannucci EL et al. (2013) No association between garlic intake and risk of colorectal cancer. Cancer Epidemiol 37, 152-155.

36. Grimes DA \& Schulz KF (2002) Bias and causal associations in observational research. Lancet 359, 248-252.

37. Win AK, Macinnis RJ, Hopper JL et al. (2012) Risk prediction models for colorectal cancer: a review. Cancer Epidemiol Biomarkers Prev 21, 398-410.

38. Zhu B, Zou L, Qi L et al. (2014) Allium vegetables and garlic supplements do not reduce risk of colorectal cancer, based on meta-analysis of prospective studies. Clin Gastroenterol Hepatol 12, 1991-2001.

39. Zhou Y, Zhuang W, Hu W et al. (2011) Consumption of large amounts of Allium vegetables reduces risk for gastric cancer in a meta-analysis. Gastroenterology 141, 80-89.

40. Zhou XF, Ding ZS \& Liu NB (2013) Allium vegetables and risk of prostate cancer: evidence from 132,192 subjects. Asian Pac J Cancer Prev 14, 4131-4134.

41. Antony ML \& Singh SV (2011) Molecular mechanisms and targets of cancer chemoprevention by garlic-derived bioactive compound diallyl trisulfide. Indian J Exp Biol 49, 805-816. 